\title{
Emission Fourier transform spectroscopy for remote sensing of the Earth's atmosphere
}

\author{
Giovanni Bianchini, Ugo Cortesi and Bruno Carli \\ Istituto di Ricerca sulle Onde Elettromagnetiche «Nello Carrara», Firenze, Italy
}

\begin{abstract}
Fourier transform emission spectroscopy can make an important contribution in the observation of the Earth's atmosphere and in the investigation of atmospheric physics and chemistry. In this paper, we report the measurement performances and the result obtained by a Fourier transform spectrometer, named SAFIRE-A (Spectroscopy of the Atmosphere using Far Infrared Emission - Airborne), operating in the far infrared spectral region from limb sounding observation of the atmospheric emission from a high altitude aircraft. After a short review of the advantages of FTS emission limb sounding in comparison with competing techniques and observation geometries, a detailed description of the instrument is given with the result obtained during the APE-GAIA (Airborne Polar Experiment - Geophysica Aircraft In Antarctica) field campaign carried out over the Antarctic Peninsula.
\end{abstract}

Key words Fourier transform spectroscopy-emission spectroscopy - Earth observation - stratospheric chemistry

\section{Emission spectroscopy of the atmosphere}

A number of chemical, dynamic and radiative processes affecting the physical structure and the composition of the Earth's atmosphere, can be investigated by measuring spontaneous thermal emission of atmospheric molecules. Emission spectroscopy benefits from unique advantages in comparison with other remote sensing methods (Carli and Carlotti, 1992; Carli et al.,1992). Being a passive technique, it does not introduce perturbations in the observed air masses and does not require energy demanding instruments. Moreover, emission measurements provide a better geographical and temporal coverage com-

Mailing address: Dr. Giovanni Bianchini, Istituto di Ricerca sulle Onde Elettromagnetiche «Nello Carrara», Via Panciatichi 64, 50127 Firenze, Italy; e-mail: gb@iroe.fi.cnr.it pared to absorption measurements. They do not need an external radiation source and can be performed continuously (both day and night) in all directions. This overcomes the poor latitude coverage of occultation measurements and makes it possible to monitor several key processes that involve chemical species with a diurnal cycle. On the other hand, observations of the signal emitted by atmospheric molecules are only possible within the frequency range with significant thermal emission from the atmosphere; this includes the middle/far infrared and the millimetre-wave spectral regions. The boundaries of the operative range of emission measurements are defined by a low frequency cutoff (approximately $3 \mathrm{~cm}^{-1}$ ) caused by the limits of optical instruments (to which this discussion is confined) and by a high frequency cut-off (approximately $3000 \mathrm{~cm}^{-1}$ ) due to the low number of emitted photons.

\subsection{The atmospheric emission spectrum}

The emission spectrum of the Earth's atmosphere consists of spectral features that correspond 


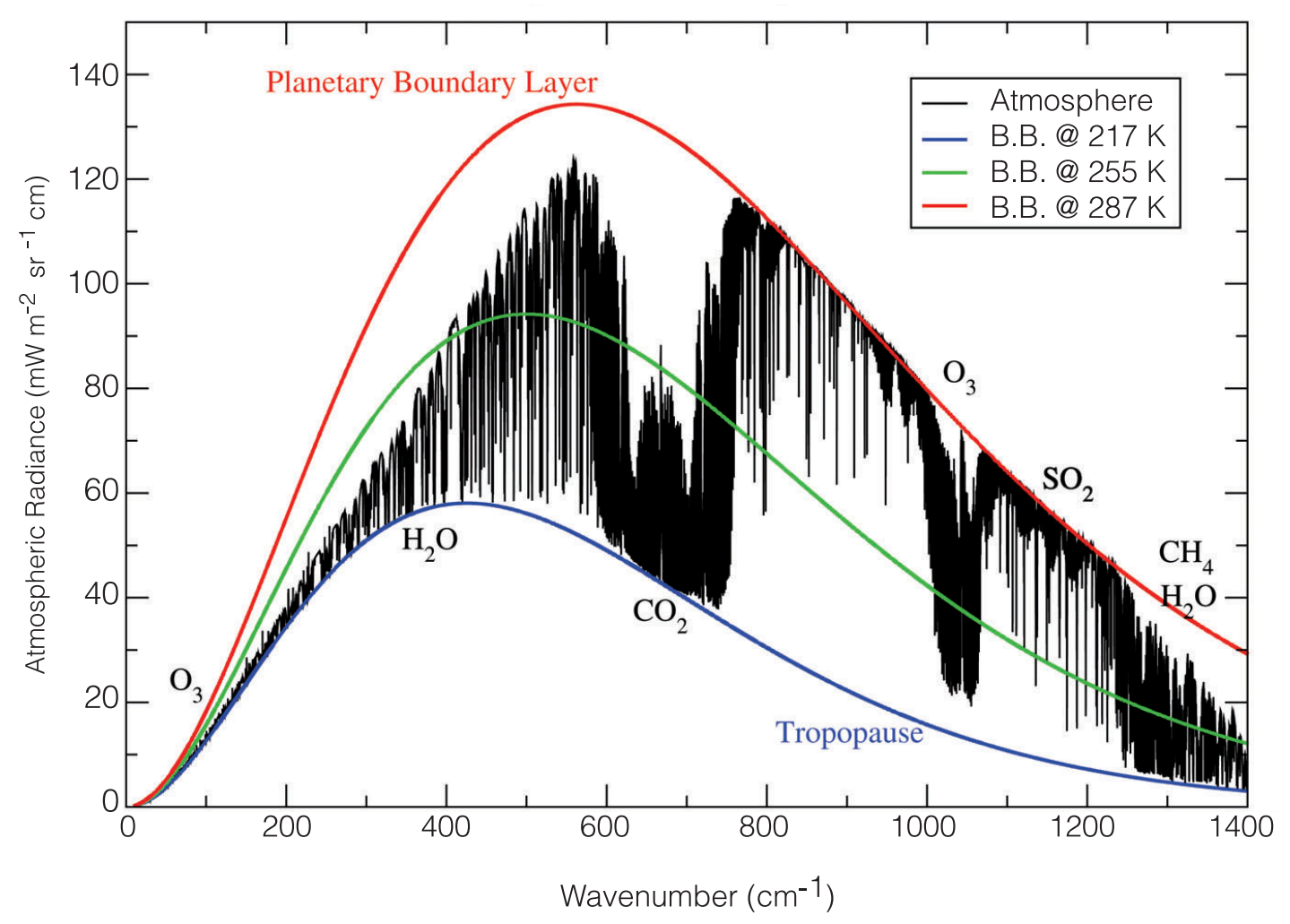

Fig. 1. Simulated emission spectrum of the Earth's atmosphere in the zenith direction.

to rotational and vibrational transitions of the atmospheric molecules (main gases and minor constituents). Pure rotational bands are associated with the millimetre $\left(3-30 \mathrm{~cm}^{-1}\right)$ and the Far Infrared (FIR, 30-300 $\mathrm{cm}^{-1}$ ) part of the spectrum, vibration bands to the Middle Infrared Region (MIR, 300-3000 $\mathrm{cm}^{-1}$ ). The intensity envelope is given by the spectral distribution of a blackbody at the temperature of the emitting air masses (typically $250 \mathrm{~K}$ in the stratosphere), as shown in fig. 1.

The dependence of the Planck function $B(\sigma)$ on temperature can be expressed as a function of wavenumber $\sigma$ according to the equation $B(\sigma)=$ $c(\sigma)\left(T / T_{0}\right)^{n(\sigma)}$. If we plot the exponent $n(\sigma)$ versus the wavenumber $\sigma$ we notice as this exponent is equal to 1 at low frequencies $\left(\sigma \ll 170 \mathrm{~cm}^{-1}\right)$ and increases greatly as the frequency is increased above that value (fig. 2). The emission in the MIR is, therefore, very sensitive to atmospheric temperature.

Temperature profiles can be retrieved with high accuracy by measuring the emission

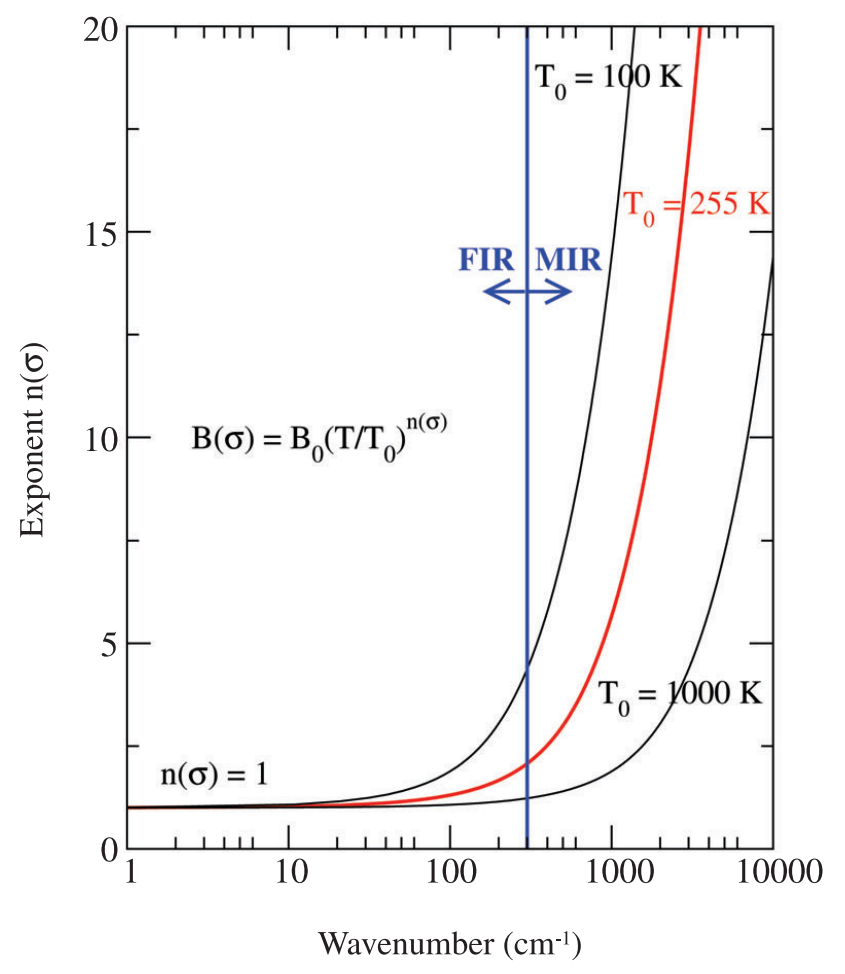

Fig. 2. Temperature dependence of the Planck function. 
spectrum of a species with well know volume mixing ratio distribution. Conversely, a very good knowledge of the temperature profile is required for retrieving minor constituents concentration from emission measurements in the MIR. From this perspective, measurements in the far infrared are relatively easier and can take advantage also from further aspects such as the freedom from scattering due to aerosols and cloud particles.

The complementarity of emission measurements in the MIR and FIR region can also be recognised in the coverage of the chemical species. Apart from some main gases (for instance, water vapour and ozone), that have ubiquitous spectra in the infrared region, in the middle infrared it is possible to measure almost the entire nitrogen family, including the source gas $\mathrm{N}_{2} \mathrm{O}$ and the important reservoir species $\mathrm{ClONO}_{2}$ with the sole exception of $\mathrm{NO}_{3}$. The detection of several compounds, that cannot be measured in the FIR, is also relatively easy in the MIR: for instance $\mathrm{CO}_{2}$ and $\mathrm{CH}_{4}$ (which, having a dipole moment equal to zero, do not display a pure rotational spectrum) or the chlorofluorocarbons. FIR remote sounders have their strength in the observation of hydrogen family. $\left(\mathrm{OH}, \mathrm{HO}_{2}, \mathrm{H}_{2} \mathrm{O}_{2}\right)$, but can also measure chlorine species ( $\mathrm{HCl}, \mathrm{HOCl}$ and $\mathrm{ClO})$, bromine species ( $\mathrm{HBr}, \mathrm{HOBr}$ and $\mathrm{BrO})$ and, even if with less sensitivity than the MIR, a few compounds of the nitrogen family $\left(\mathrm{N}_{2} \mathrm{O}, \mathrm{HNO}_{3}\right)$.

\subsection{Observing geometries and techniques}

Different combinations of the observation platform (ground-based stations, high altitude platforms or satellites) and viewing geometry (vertical sounding or limb sounding) can be chosen, in principle, for remote-sensing of the Earth's atmosphere.

Vertical sounding can be performed either from the ground, looking at the zenith, or from high altitude and space-borne platforms in nadir looking mode. Limb sounding measurements are possible from stratospheric balloons and aircraft, as well as from space, and generally provide a much higher vertical resolution with respect to vertical soundings. Each of these options offers specific advantages and disadvantages, as shown in table I, with limb sounding being particularly well suited for the study of upper tropospheric and lower stratospheric chemistry.

Despite their wider spatial and temporal coverage, limb sounding observations from space have limited capability of capturing small scale features in the lowest altitude range. In fact, balloon and airborne instruments are usually the best choice for detailed investigations of the physical and chemical structure of the upper troposphere and lowermost stratosphere and for the study of local phenomena. The operating altitude of large stratospheric balloons (approximately $40 \mathrm{~km}$ ) is higher than the ceiling of highflying aircraft $(20 \mathrm{~km})$ and offers a better chance for limb scanning. On the other hand, the mobility and manoeuvrability of the aircraft, as well as the possibility of performing several flights within a relatively short time period cannot be obtained with balloon-borne instrumentation. Moreover, a large scientific payload can be accommodated aboard an aircraft, making possible simultaneous measurements of several chemical compounds and other atmospheric parameters.

Table I. Comparison between different observing platforms and geometries.

\begin{tabular}{ccc}
\hline \hline Geometry & Platform & Notes \\
\hline Zenith & Ground based & Only local measurements near station \\
Nadir & Space platform & Global cover, low horizontal/vertical resolution \\
Nadir & Aircraft & High horizontal resolution, low vertical resolution \\
Limb & Space platform & Global cover, low horizontal resolution \\
Limb & Aircraft/balloon & Optimal horizontal/vertical resolution for localphenomena \\
\hline
\end{tabular}




\subsection{Limb sounding observation scheme}

The concept of limb sounding observation geometry is illustrated in fig. 3a: the instrument aboard a high altitude platform or satellite observes radiation emitted or absorbed by the air masses along a line of sight tangent to an atmospheric layer. The main contribution to the observed signal, as shown in the figure, comes from the lowest atmospheric layer intersected by the line of sight, thus making limb sounding measurements highly selective in the vertical direction.

The main advantages of limb geometry compared to the vertical sounding are:

1) The absorbing (or emitting) path is larger and delivers detectable signals also from very weak absorbers (or emitters).

2) Emission measurements are made with deep space, rather than warm Earth's surface, at the end of the line of sight.

3) High vertical resolution is obtained, typically $2-3 \mathrm{~km}$, with the possibility of scanning the line of sight across the atmospheric layers and retrieving the vertical concentration profiles from a sequence of measurements performed at different limb angles.

A drawback of a limb sounding measurement is the poor horizontal resolution in the direction perpendicular to the aircraft heading, typically about $500 \mathrm{~km}$, due to the very long distances travelled by the line of sight in the atmosphere. The resolution along the flight path, as shown in fig. $3 \mathrm{~b}$, is instead given by the product of limb scanning sequence acquisition time and aircraft ground speed.

\subsection{Fourier transform spectroscopy for Earth observation}

A wealth of spectroscopic techniques and instruments is available nowadays for passive emission sounding of the Earth's atmosphere. Among these, Fourier Transform Spectroscopy (FTS) still represents the preferred option in several studies, whose measurement requirements are only partially satisfied by competing techniques. In fact, Fourier transform spectrometers provide a specific combination of attributes that can efficiently match different observing needs, such as those imposed, for instance, by investigation of upper tropospheric and lower stratospheric chemistry. The classical advantages of FTS can be fully exploited when applied to atmospheric measurements: the detection of very weak signals emitted (or absorbed) by atmospheric molecules and their acquisition with adequate temporal and spatial resolution become possible only because of the gain introduced by the throughput and the multiplex advantage (Chamberlain, 1978). Furthermore, the capability of resolving spectral features due to minor middle atmospheric constituents is granted by the high spectral resolution provided by FTS measurements, which can easily meet the typical requirement for a resolving power better than 10000 (corresponding to a limit spectral resolution of 0.1 $\mathrm{cm}^{-1}$ in the MIR and of $0.01 \mathrm{~cm}^{-1}$ in the FIR). This results in a fundamental advantage when coupled with the broadband operation potential of Fourier transform instruments, that permits the simultaneous measurement of a large suite of chemical compounds.

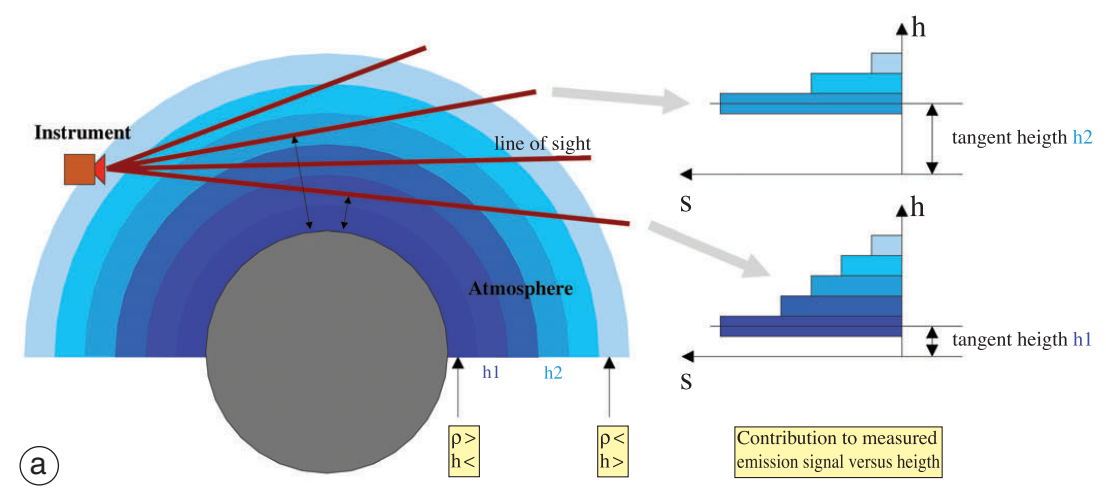

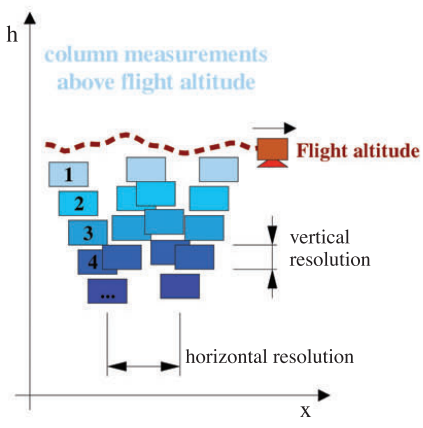

(b)

Fig. 3a,b. The limb sounding observation geometry: a) vertical resolution; b) horizontal resolution. 


\section{The SAFIRE-A FTS spectrometer}

SAFIRE-A is a particular implementation of the Martin-Puplett (Martin and Puplett, 1969) polarising FT spectrometer specially designed for airborne operation (Carli et al., 1969). The principal instrumental characteristics are summarised in table II. Optical path folding and a misalignment-compensating (Carli, 1989) optical scheme have permitted to enclose the whole instrument, including optics, control and data storage electronics and cryogenic detectors in a compact package suitable for integration on the Russian M-55 Geophysica high altitude aircraft (fig. 4).

The M-55 Geophysica, a modified version of a former reconnaissance aircraft operated by the Myasishchev Design Bureau, is, together with the American ER-2, the only platform available in the world for stratospheric research. It is a dual turbofan single-seater aircraft capable of flying day and night in all weather conditions. The platform can operate at a ceiling altitude of 21 $\mathrm{km}$ for about $5 \mathrm{~h}$. The maximum cruise ground speed is about $750 \mathrm{~km} / \mathrm{h}$ and the operative radius at $17 \mathrm{~km}$ altitude is approximately $3500 \mathrm{~km}$. The M-55 Geophysica has unique capabilities for carrying large scientific instruments: it can accommodate a payload with a weight up to a maximum of $1500 \mathrm{~kg}$ and with a total volume of up to $12 \mathrm{~m}^{3}$. A large reserve of electric power (60 kW at $115 \mathrm{VAC}$ and $3 \mathrm{~kW}$ at $27.5 \mathrm{VDC}$ ) also makes it possible to embark energy demanding instruments (e.g., high power lidars).

\subsection{Instrument optical layout}

As shown in fig. 5, the SAFIRE-A instrument is made of four main parts, the input optics system, the interferometer, the Cold Optics and Detector Module (CODM) including output optics and detectors, and the electronic control and acquisition system.

\subsubsection{Input optics}

A schematic representing the input optics system is shown in fig. 6. Atmospheric emission is collected through a pointing mirror which is controlled in position using the roll angle information provided by the aircraft navigation system with an accuracy of about 20 arcsec. A calibration system, located in the focus of the input telescope, permits us to choose the reference source from two calibration blackbodies at different temperatures.

Table II. Characteristics of the SAFIRE-A Fourier transform spectrometer.

\begin{tabular}{lc}
\hline \hline \multicolumn{1}{c}{ Parameter } & Value \\
\hline Instrument type & Polarising FT spectrometer \\
Dimensions & $1800 \times 880 \times 650 \mathrm{~mm}$ \\
Weight & $387 \mathrm{~kg}$ \\
Interferogram acquisition time & $12,24,48,96 \mathrm{~s}$ \\
Number of detection channels & 2 \\
Operating spectral range & $10-250 \mathrm{~cm}^{-1}$ \\
Maximum spectral resolution & $0.004 \mathrm{~cm}^{-1}$ \\
Vertical resolution & About $1.5 \mathrm{~km}^{\circ}$ \\
Vertical field of view & $0.57^{\circ}$ \\
Spectral signal-to-moise ratio & $>500$ \\
Observation technique & Limb-sounding emission \\
\hline
\end{tabular}




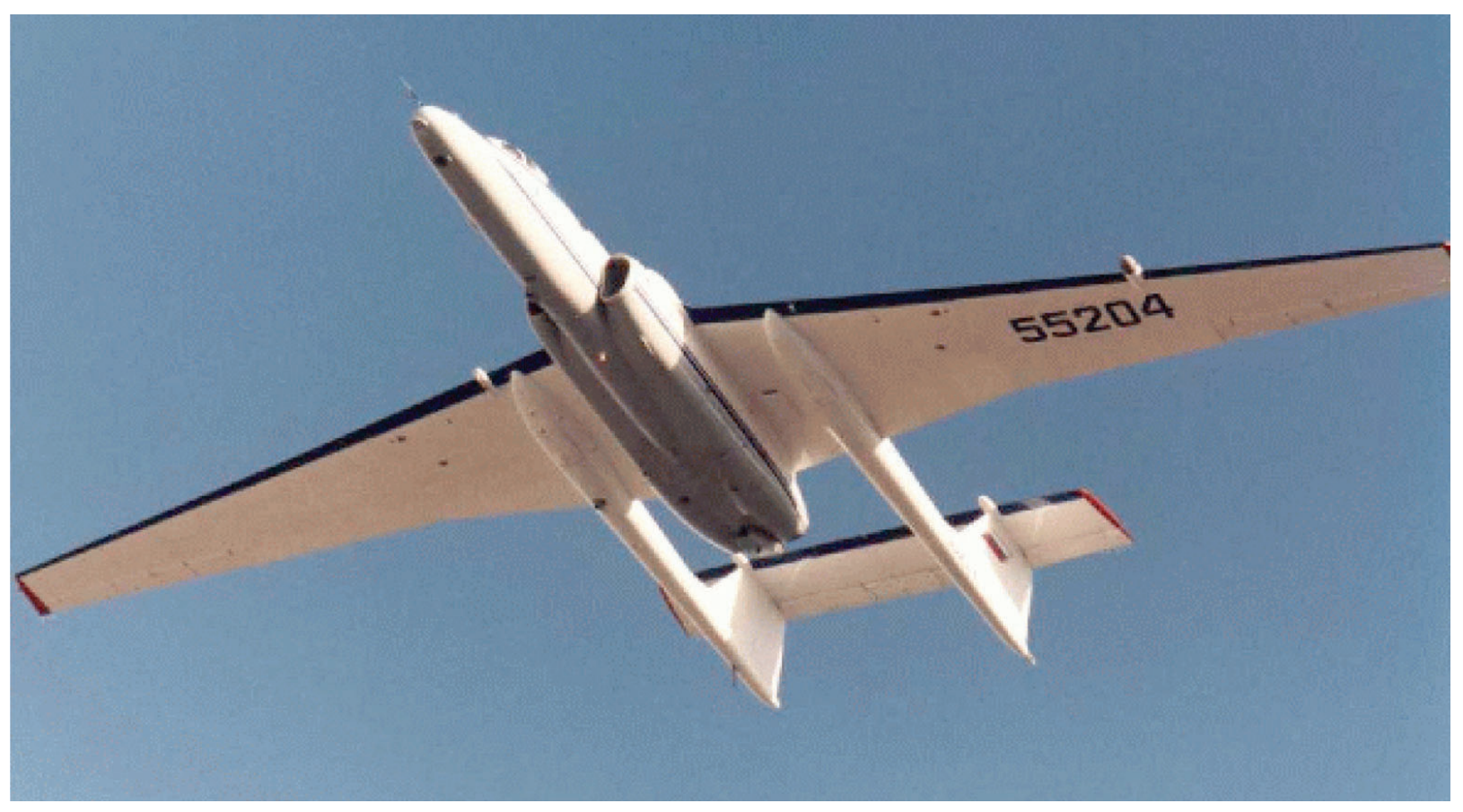

Fig. 4. The Myasischev M-55 Geophysica stratospheric aircraft.

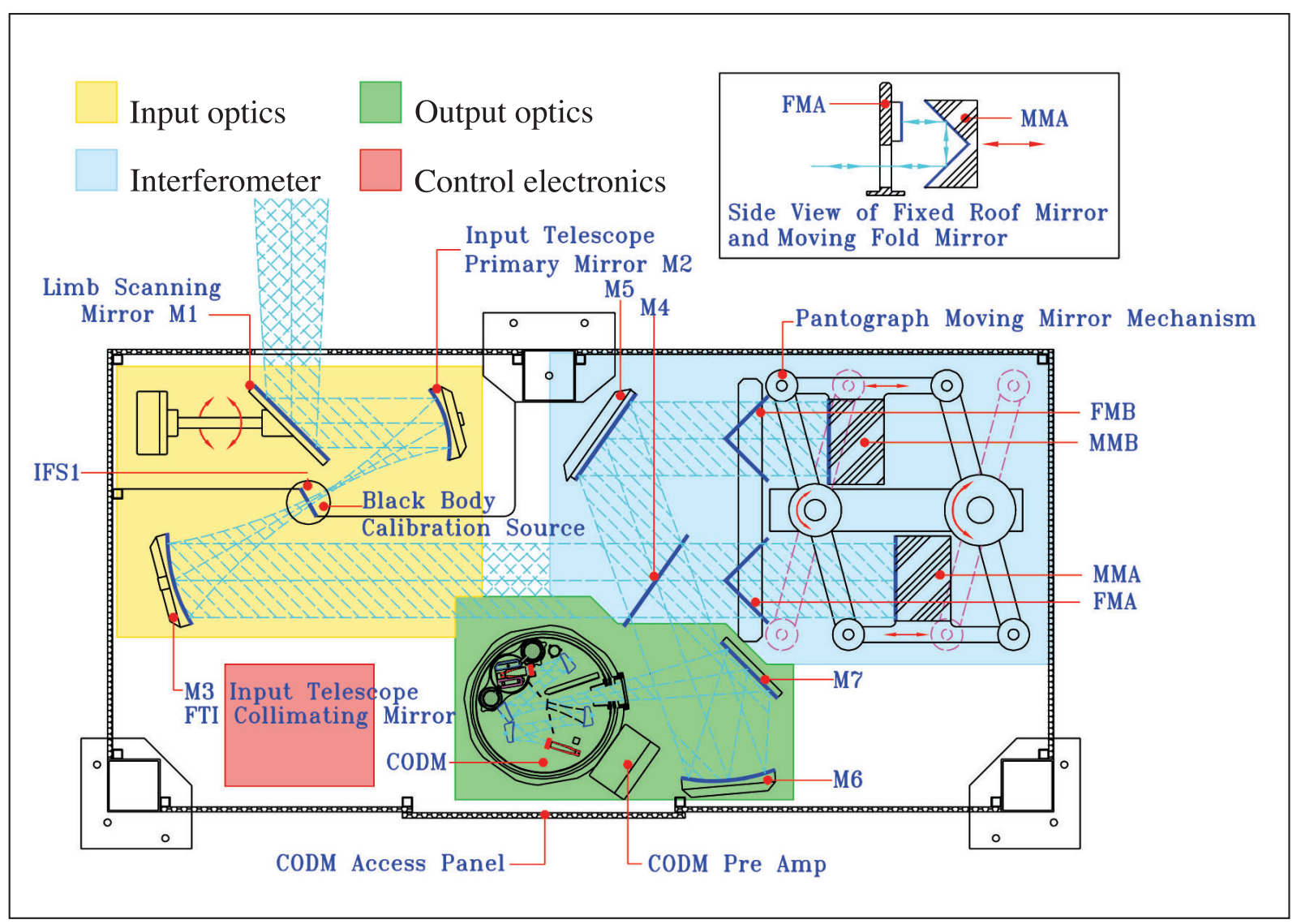

Fig. 5. The SAFIRE-A spectrometer, optical layout. 


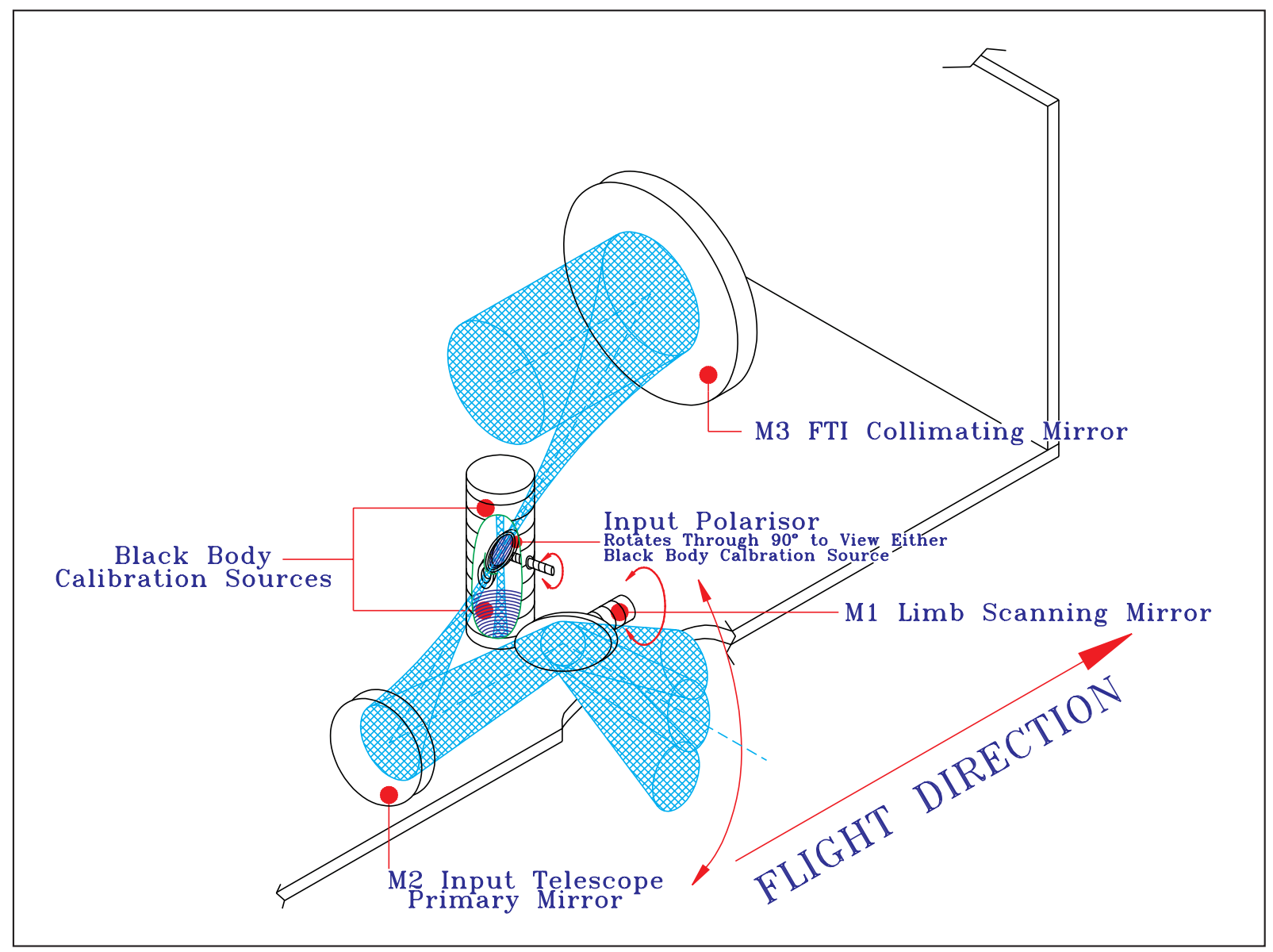

Fig. 6. Schematic of the SAFIRE-A input optics.

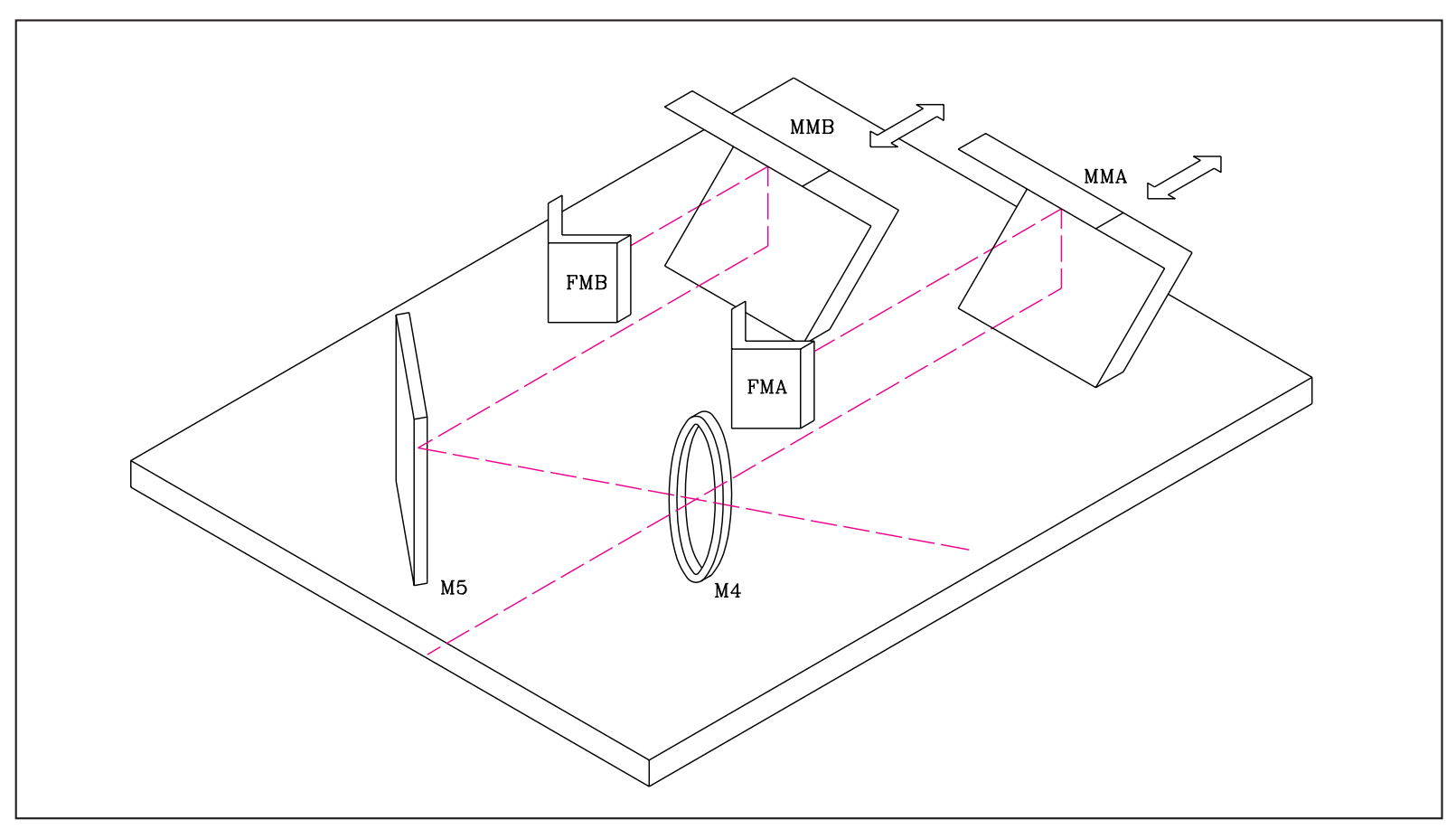

Fig. 7. The SAFIRE-A FIR Interferometer. 


\subsubsection{FIR interferometer}

The FIR interferometer (fig. 7) is based on a double parallelogram scheme that through a folding of the optical path and the simultaneous movement of both mirrors provide a maximum Optical Path Difference (OPD) between the two arms of the interferometer equal to $1.25 \mathrm{~m}$, containing the optics in a cube with a side of about $0.5 \mathrm{~m}$. Accurate measurement of OPD is obtained through a reference laser interferometer based on a frequency-stabilised diode laser source and following the same optical path of the far infrared radiation (Bianchini et al., 2000).

\subsubsection{Cold optics and detector module}

The CODM encloses output optics, including pupils and field stops, blocking filters and collecting optics, and the two detectors corresponding to the two acquisition channels. All these components are mounted on the cold plate of a liquid helium cryostat, and allow the full exploitation of photon-noise limited detectors. Two different kinds of infrared detectors are used on SAFIRE-A: photoconductive detectors operating at $4 \mathrm{~K}$ cover spectral intervals extending from 50 to $250 \mathrm{~cm}^{-1}$, and bolometric detectors operating at $0.3 \mathrm{~K}$ to cover the 10 to $50 \mathrm{~cm}^{-1}$ region. In this case, a closed circuit ${ }^{3} \mathrm{He}$ cooling stage is mounted on the cold plate of the cryostat. A schematic drawing of the optical layout of the CODM and a photograph of the system are shown in fig. 8.

\subsubsection{Acquisition and control electronics}

The sequence of operations necessary to data acquisition (pointing mirror control, reference source changes, moving mirror scan) are controlled by an on board computer.

Atmospheric emission data, in the form of raw interferograms, are stored by the on board computer on an array of mirrored hard disks in a pressurized casing, along with housekeeping information useful to monitor instrument status.

Figure 9a,b shows two photographs of the instrument. In fig. 9a most of the EMI shielding enclosing the instrument is removed to show internal parts, in fig. $9 \mathrm{~b}$ the instrument is installed on the M-55 Geophysica platform.

\subsection{Instrument performances}

As part of the effort for processing and evaluation of data collected by SAFIRE-A during the APE-GAIA campaign, accurate checks of data quality were carried out for diagnostic purposes and a new assessment of measured instrument performances was obtained. Taking into account that important modifications were recently made to the spectrometer with the aim of optimising the instrumental set-up in view of the Antarctic mission, this kind of analysis attained the twofold objective of: verifying the actual improvements in instrument performances, and obtaining a full characterisation of the optimised instrument configuration.

The Instrument Line Shape (ILS) was determined by averaging multiple spectra recorded with the same acquisition parameters and fitting an isolated spectral line, having a spectral width much lower than instrumental resolution, with the theoretical expression for the FTS lineshape: $\operatorname{ILS}(\sigma)=\operatorname{sinc}\left(\left(\sigma-\sigma_{0}\right) / \Delta \sigma\right) . \sigma_{0}$ is the centre frequency of the spectral line and $\Delta \sigma$ is the spectral resolution. The results are shown in fig. 10. The obtained value of $0.004 \mathrm{~cm}^{-1}$ for $\Delta \sigma$ is in good agreement with the resolution determined by the maximum OPD, showing that the mechanisms limiting the resolution (like field of view effects or instrumental vignetting) have negligible effects.

The frequency calibration stability of SAFIRE-A depends on the reference laser interferometer (Bianchini et al., 2000), that is one of the instrument subsystems that received the most significant modifications and improvements. The reference laser stability was measured by fitting a single line in different spectra selected at regular time intervals throughout a flight (about $6 \mathrm{~h}$ of duration). A laser frequency variation $\Delta v$ will induce a corresponding line centre variation $\Delta \sigma$ such that $\Delta v / v_{L}=\Delta \sigma / \sigma_{0}$, where $v_{L}$ is the laser frequency, and $\sigma_{0}$ the centre of the atmospheric spectrum line. Figure 11 shows the line centre values. Considering that the expected behaviour 

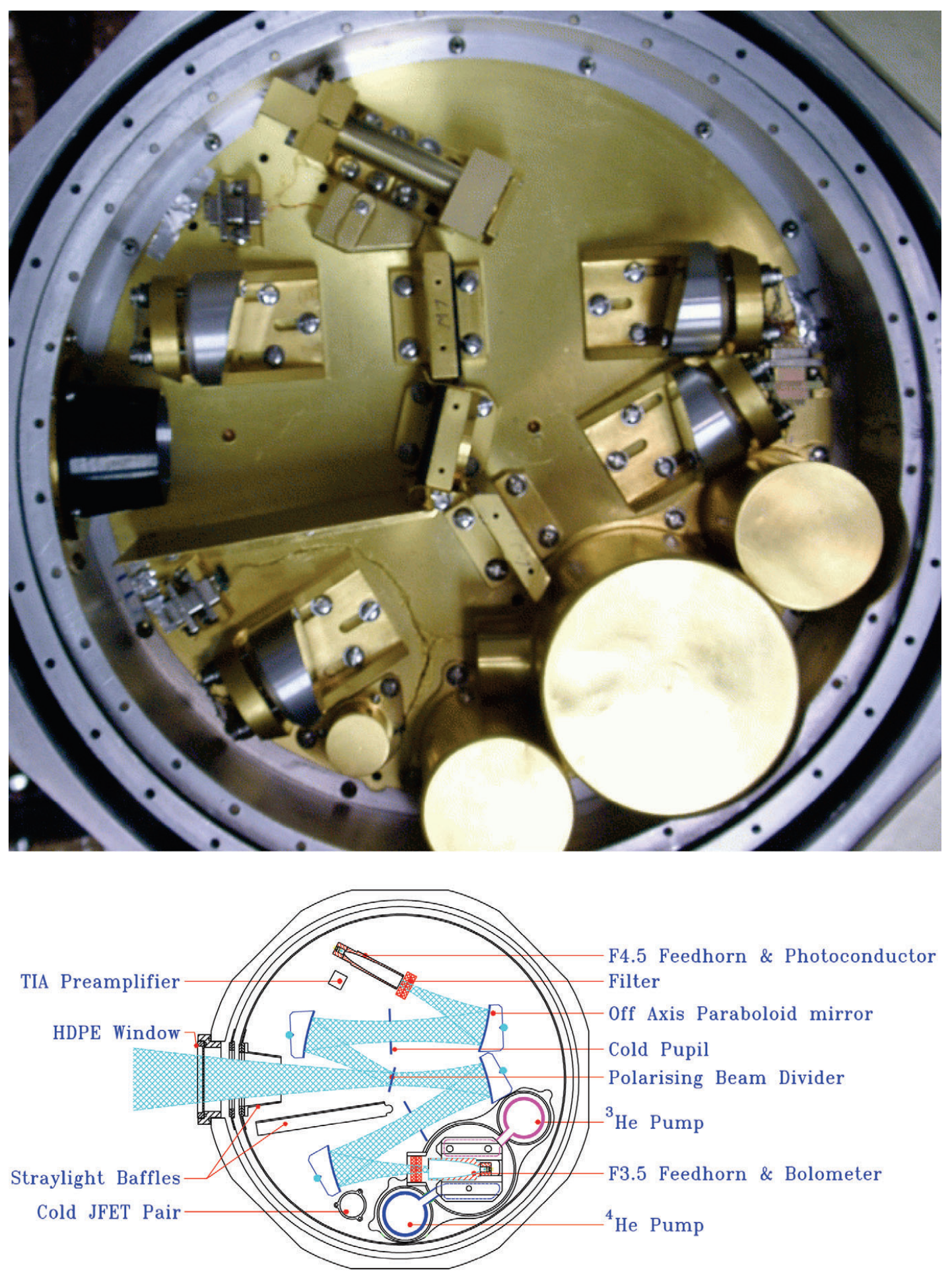

Fig. 8. The Cold Optics and Detector Module (CODM).

is a slow drift due to thermal effects in the laser control electronics, a linear fit is superimposed to the data points. The obtained drift ratio is about $-0.6 \mathrm{ppm} / \mathrm{h}$, well inside instrumental re- quirements of less than $6 \mathrm{ppm} / \mathrm{h}$ (Martin and Puplett, 1969).

Instrumental responsivity may also vary between two subsequent calibration operations 


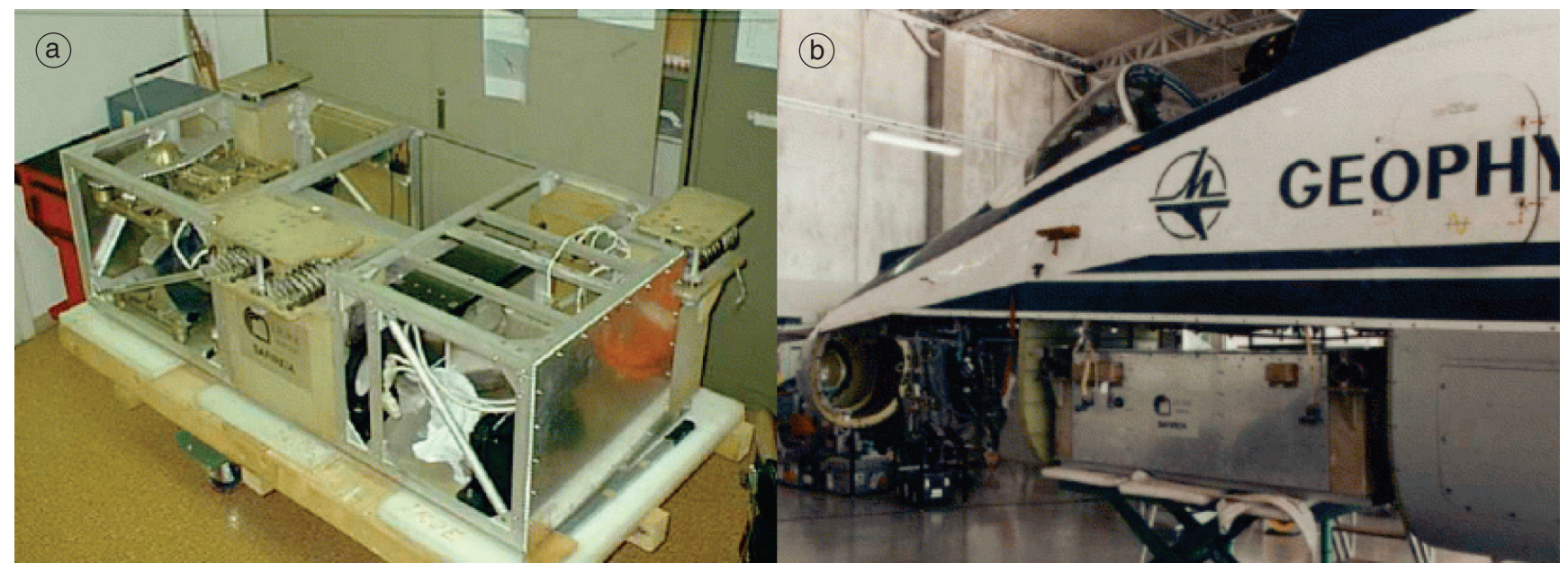

Fig. 9a,b. Photographs of the SAFIRE-A instrument: a) instrument ready for laboratory operations; b) instrument installed on the M-55 Geophysica.

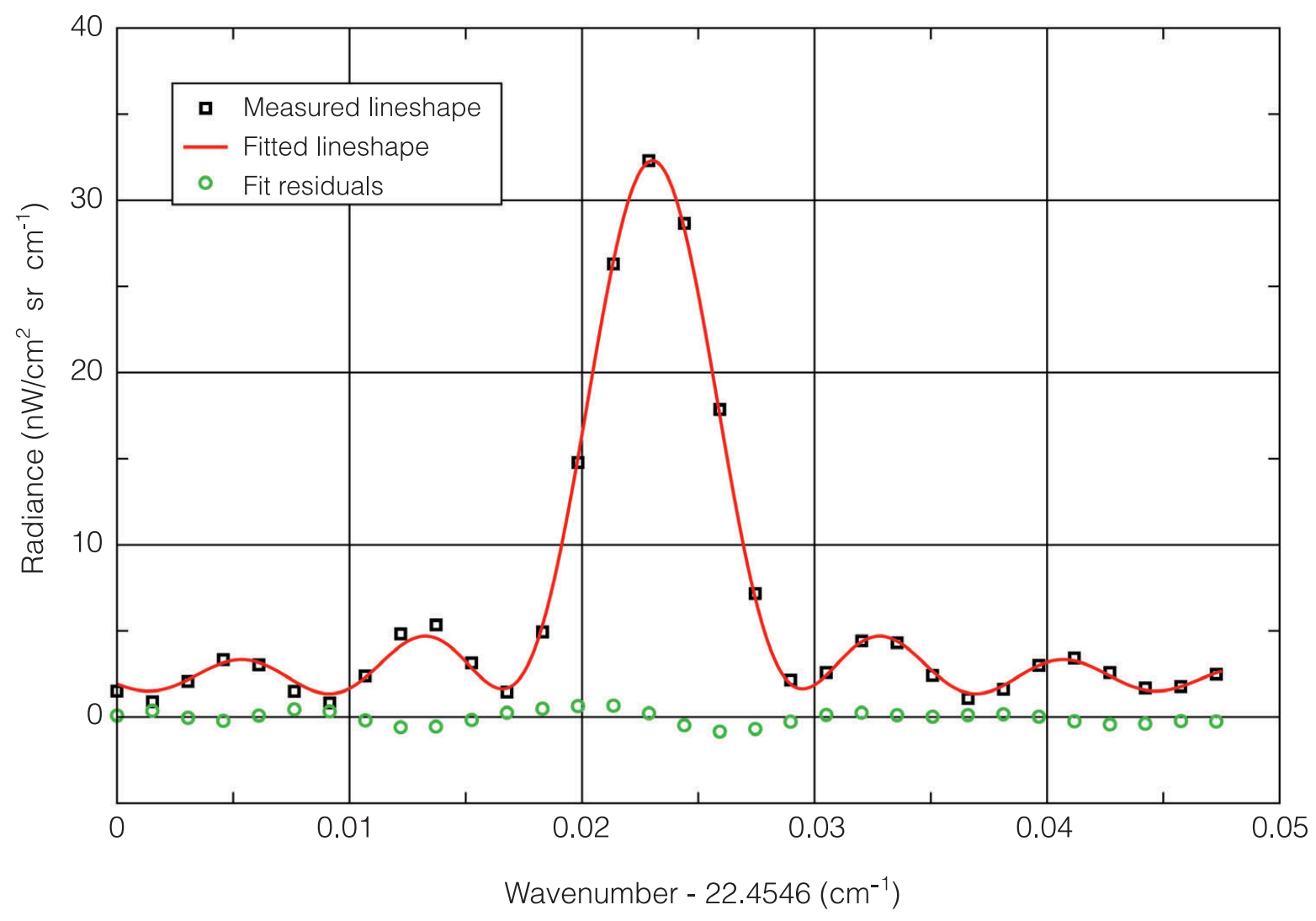

Fig. 10. Instrumental Line Shape (ILS) of the SAFIRE-A spectrometer (flight 12.10.1999).

occurring every half an hour. As a consequence, amplitude calibration errors are introduced in the measured spectra. Figure 12 shows two instrumental response functions, measured during the first and the second parts of a flight. From this comparison we can determine that responsivity variations on the time-scale of a few hours are below instrumental noise. 


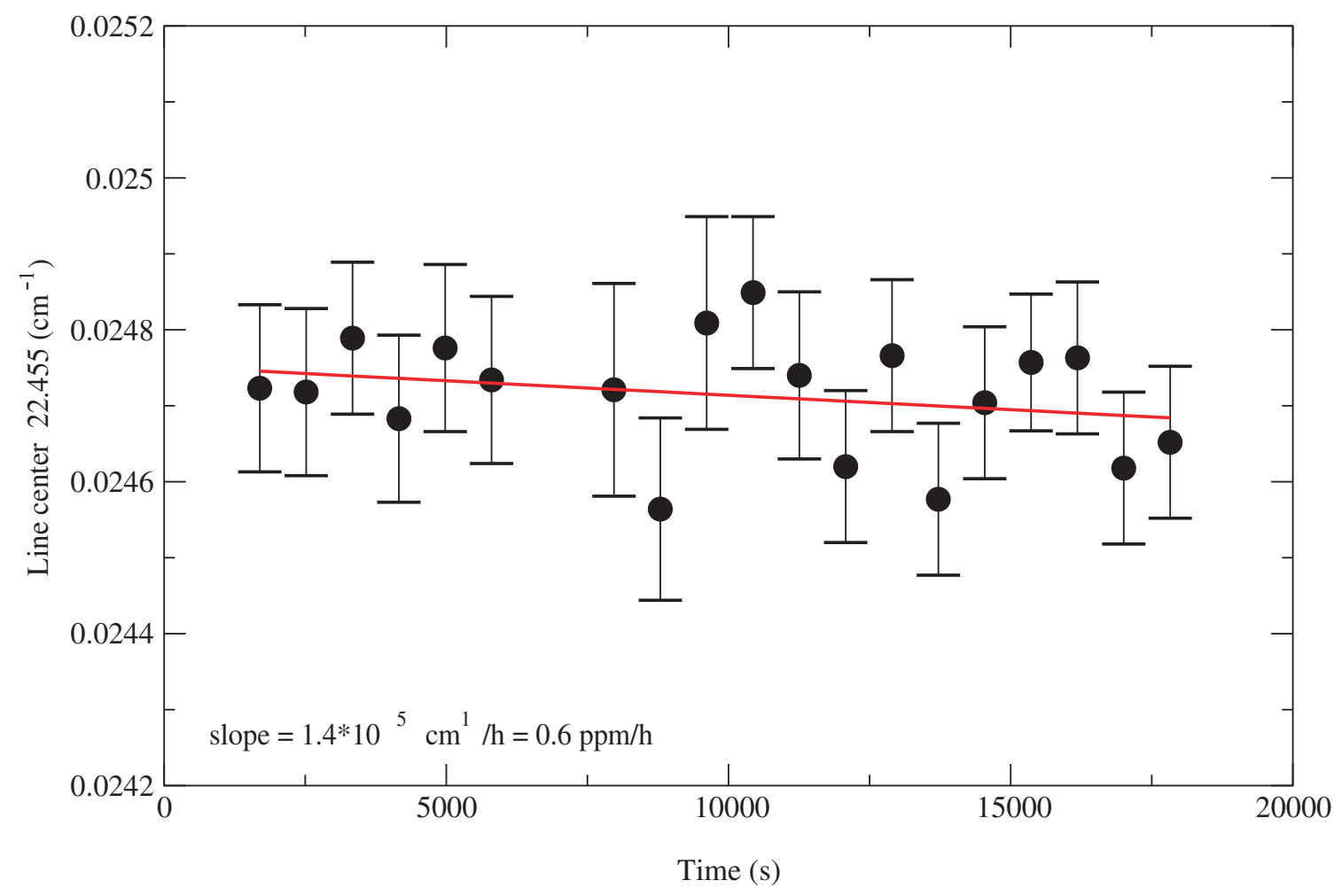

Fig. 11. Frequency stability of the SAFIRE-A laser reference system (flight 08.10.1999).

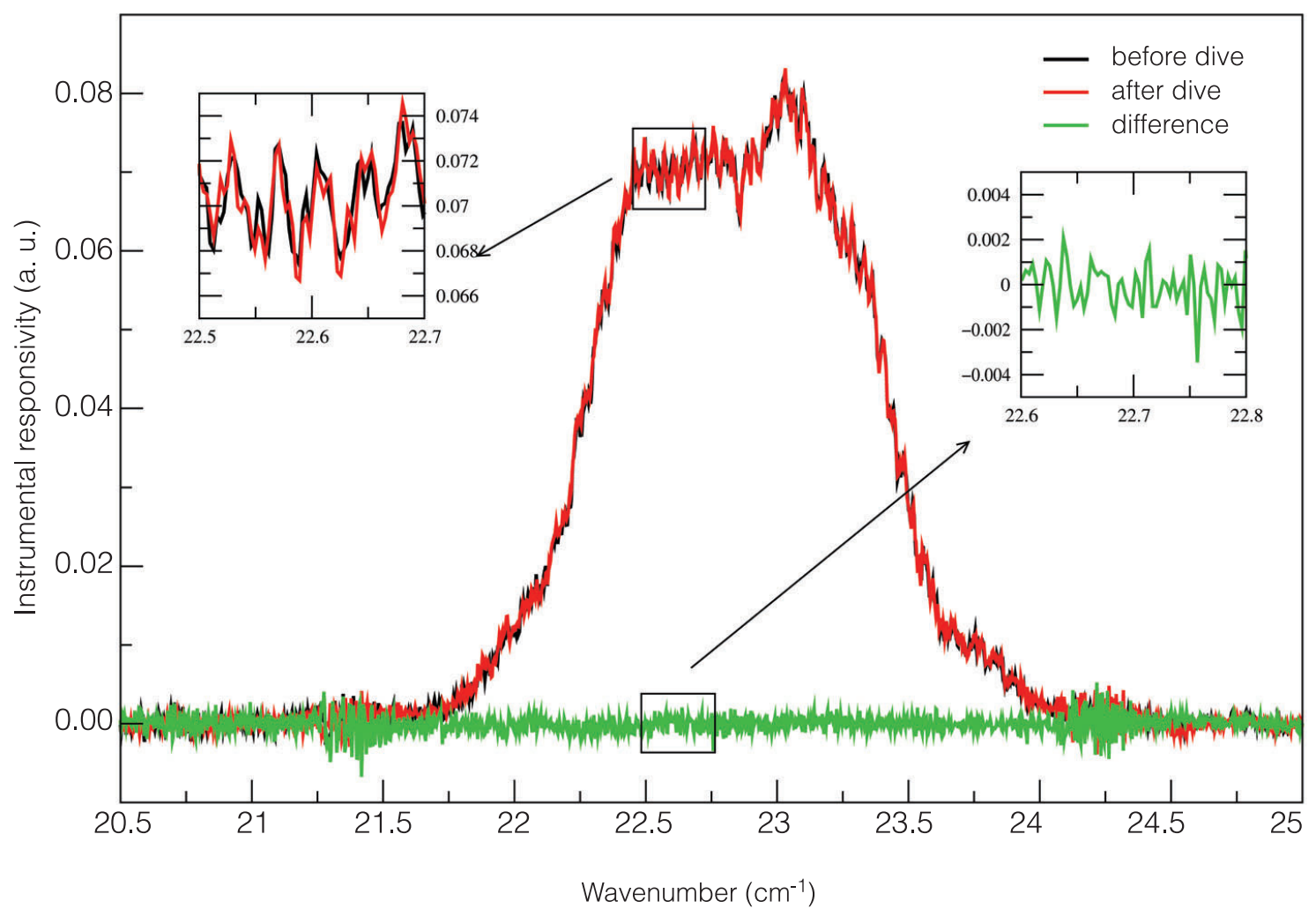

Fig. 12. Radiometric calibration stability of the SAFIRE-A spectrometer (flight 08.10.1999). 
3. SAFIRE-A measurements during the APEGAIA Antarctic campaign

A key role was played by the SAFIRE-A instrument during the APE-GAIA Antarctic campaign (Airborne Polar Experiment - Geophysica Aircraft In Antarctica) carried out in September-October 1999 from the operative base of Ushuaia, Argentina (lat. $55^{\circ} \mathrm{S}$, long. $68^{\circ} \mathrm{W}$ ) located at the southernmost tip of South America. The scientific mission, supported by the Italian National Program for Antarctic Research (Programma Nazionale di Ricerche in Antartide, PNRA), aimed at studying the upper tropospheric and lower stratospheric chemistry and transport at the boundary of the southern polar vortex during the transition period between the end of the ozone depletion and the beginning of the recovery phase. The stratospheric platform was equipped therefore with a remote-sensing and in situ payload capable of measuring both the chemical and physical properties of the polar air masses.

Five scientific flights were performed by the high altitude platform, taking off from the Ushuaia airport and heading south, towards the Palmer Peninsula (see fig. 13); trying to reach the polar vortex edge, cross the boundary and penetrate as deep as possible into the Chemically Perturbed Region (CPR).

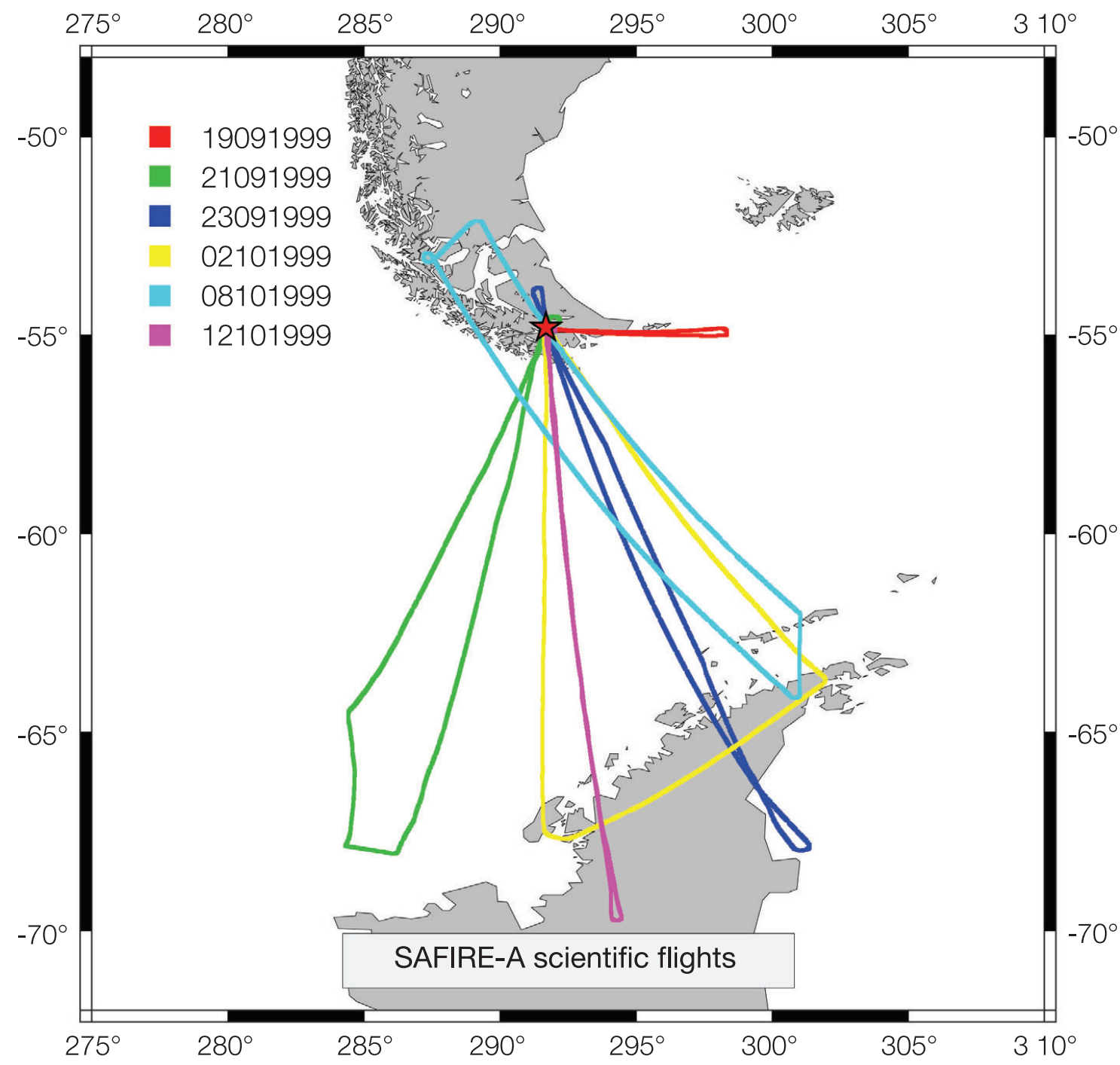

Fig. 13. Map of the APE-GAIA scientific flights routes. 
The SAFIRE-A spectrometer was flown in combination with the MIPAS-STR (Michelason Interferometer for Passive Atmospheric Sounding STRatospheric aircraft, IMK-FZK, Germany, Piesch et al., 1996), thus providing the first opportunity for simultaneous measurements of two limb sounding instruments operating respectively in the far infrared and middle infrared spectral regions (Fisher, 1992). As a consequence, it was possible to attain a comprehensive coverage of chemical compounds, including the main reactive species and reservoirs of the most important families responsible for catalytic ozone removal $\left(\mathrm{Cl}_{\mathrm{y}}, \mathrm{NO}_{\mathrm{y}}, \mathrm{HO}_{\mathrm{x}}\right.$, etc. $)$ in the Antarctic stratosphere. During the APE-GAIA campaign, the SAFIRE-A spectrometer was equipped with a detector module including a long-wavelength detection channel operating in the $22-23.5 \mathrm{~cm}^{-1}$ spectral window, and a short wavelength channel operating at $123-125 \mathrm{~cm}^{-1}$. The detected species in the long-wavelength window are $\mathrm{O}_{3}, \mathrm{ClO}$,
$\mathrm{N}_{2} \mathrm{O}$ and $\mathrm{HNO}_{3}$, the short wavelength window provides measurements for $\mathrm{HCl}$ and $\mathrm{H}_{2} \mathrm{O}$.

\subsection{Observing geometry}

The observation strategy adopted by the SAFIRE-A instrument during the scientific flights over the Antarctic Peninsula was based on a series of limb scanning sequences of 10 emission spectra. During each sequence the atmosphere was vertically sampled with a vertical resolution of about $1.5 \mathrm{~km}$. A single spectrum is acquired in $30 \mathrm{~s}$, thus resulting in an acquisition time of approximately $5 \mathrm{~min}$ for the individual limb scanning sequence. Considering an aircraft average ground speed of about $700 \mathrm{~km} / \mathrm{h}$ at cruise altitude, we obtain horizontal resolution of about $50 \mathrm{~km}$ in the flight direction, corresponding to 0.5 degrees of latitude. The horizontal resolution in the direction of the instrument line of sight

SAFIRE-A

Detection unit 1 (long wavelength), channel 1

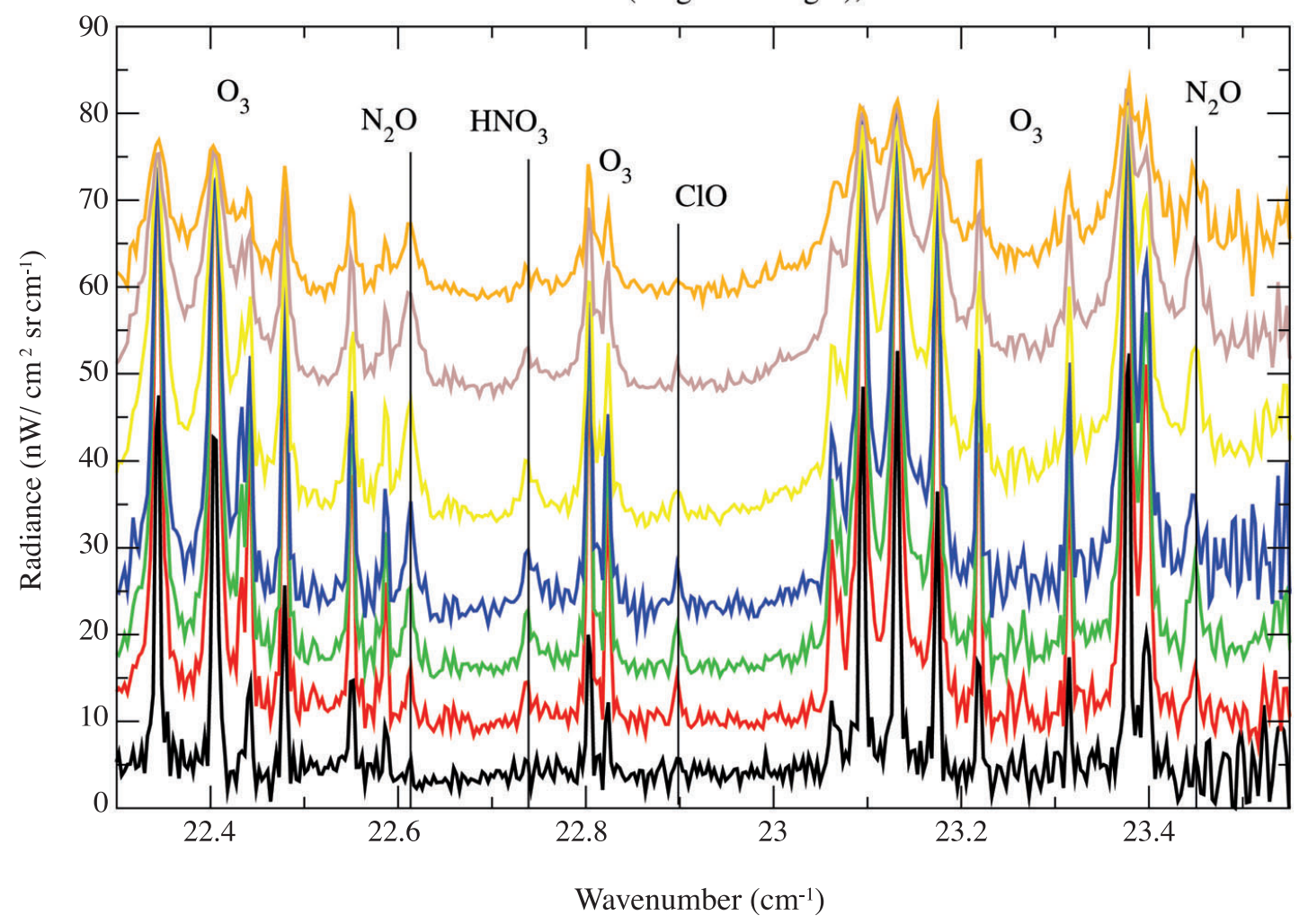

Fig. 14. Limb-scanning sequence of atmospheric emission spectra acquired in the $22-23.5 \mathrm{~cm}^{-1}$ window. Spectral features due to interesting molecular species are evidenced by vertical lines. 
SAFIRE-A

Detection unit 1 (long wavelength), channel 2

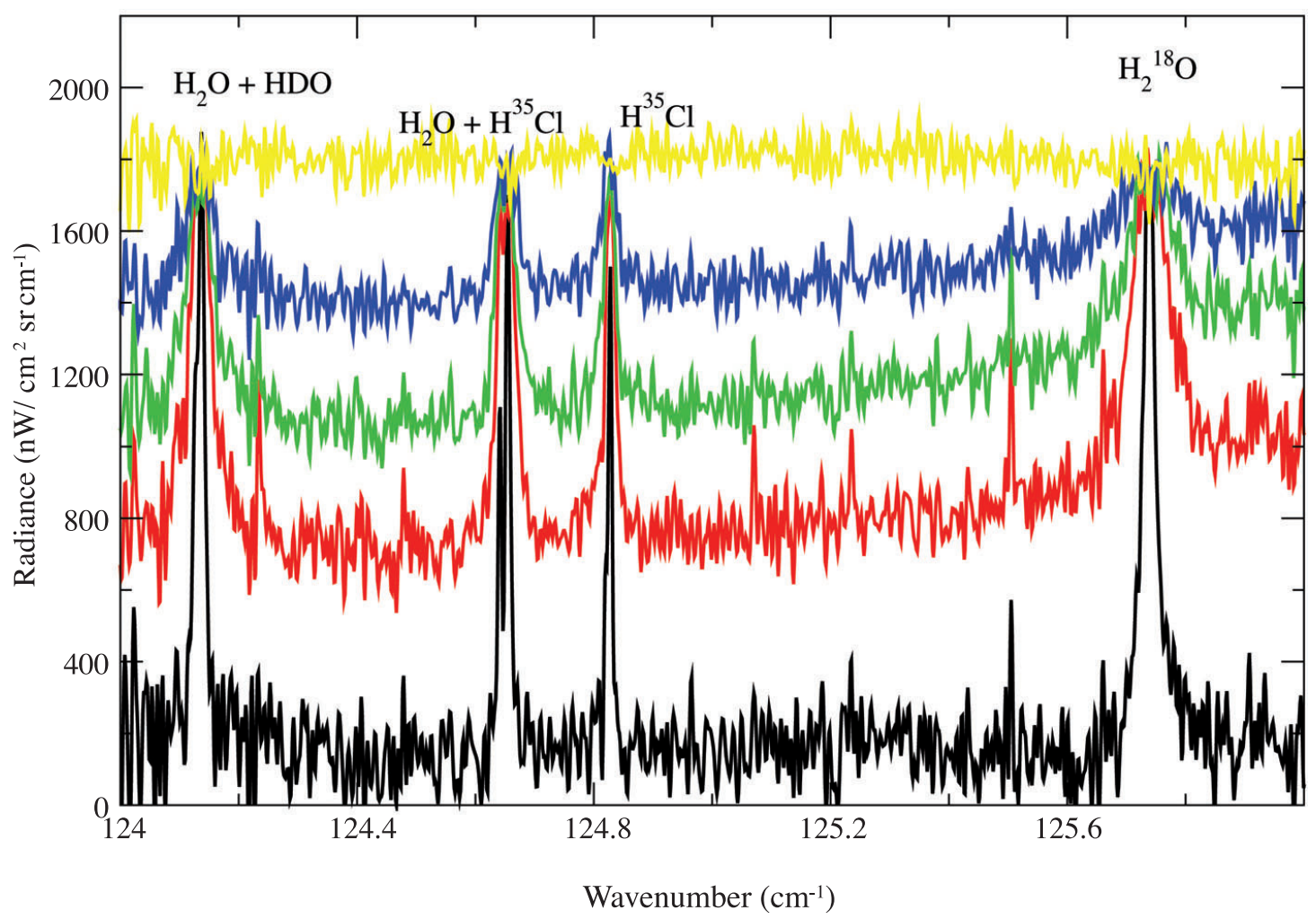

Fig. 15. Limb-scanning sequence of atmospheric emission spectra acquired in the $124-126 \mathrm{~cm}^{-1}$ window. Spectral features due to interesting molecular species are evidenced by vertical lines. Atmospheric opacity due to water vapour absorption limits analysis to upper atmospheric layers, so only limb scanning angles corresponding to upper layers are presented.

depends on the limb angle, ranging from several hundred meters for observation close to the horizontal up to several hundred kilometers for the lowest tangent altitudes.

Figure 14 shows a typical limb scanning sequence of atmospheric emission spectra in the $22-23.5 \mathrm{~cm}^{-1}$ window, where location of spectral features due to the interesting molecular species are evidenced by vertical lines. Figure 15 shows a similar plot for the $123-125 \mathrm{~cm}^{-1}$ window. In this case, only the first few limb scanning angles, corresponding to upper atmospheric layers, are presented. This is due to the greater water vapour contribution to atmospheric opacity, that prevents analysis of lower atmospheric layers. The high frequency channel was affected by very large noise because of the low efficiency at high frequencies of the free standing wire grid beam splitter that was used in order to avoid the acoustic vibrations of the substrate.

\subsection{Volume mixing ratio measurements}

The analysis of the limb scanning atmospheric measurements was performed using the global-fit approach described in Carlotti (1988). Volume Mixing Ratio (VMR) vertical profiles of the observed species from the tropopause (about $10 \mathrm{~km}$ ) to flight altitude (maximum $20 \mathrm{~km}$ ) are retrieved from the limb scanning sequences acquired along the flight path. The most interesting information from SAFIRE-A VMR vertical profiles can be obtained mapping VMR values versus altitude and aircraft ground position, reconstructing a two-dimensional representation of a slice of atmosphere, thus evidencing the vertical and geographical variability of stratospheric composition due to chemical and dynamical processes. For a typical Antarctic flight the geographical coverage extends on the latitude range from about $58^{\circ} \mathrm{S}$ to $68^{\circ} \mathrm{S}$. 


\subsection{Results obtained during the APE-GAIA mission}

The measurements performed by the SAFIREA spectrometer during the five scientific flights of the APE-GAIA mission lead to a wealth of science quality data that can be used to investigate both chemical and dynamical processes occurring in the Antarctic lower stratosphere. Some of the most interesting results are reported here, to highlight the instrument capabilities and to show how it can contribute to the study of a variety of scientific issues.

\subsubsection{Ozone chemistry}

Figure 16 shows VMR maps corresponding to two of the species detected in the longwavelength channel (ozone and $\mathrm{ClO}$ ) during the 09.23.1999 flight. In both plots the horizontal axis represents flight time in seconds UTC (Universal Time Co-ordinates). Flight altitude (superimposed to the map) is shown for reference. The flight considered consists in a first leg in which the aircraft was heading south, crossing the polar vortex edge and entering the vortex, and a second leg heading north, along the
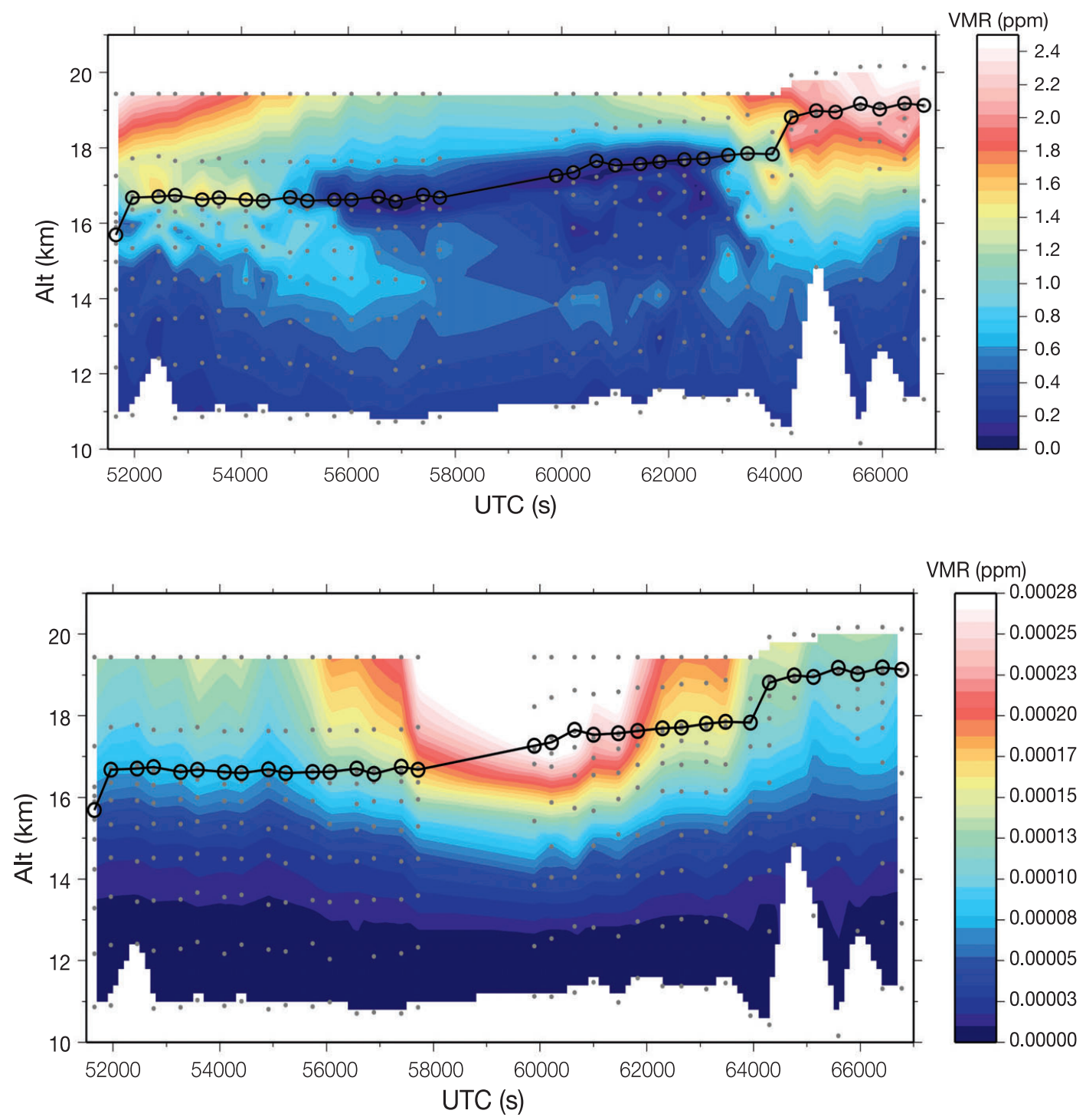

Fig. 16. Ozone (upper panel) and $\mathrm{ClO}$ (lower panel) VMR maps obtained from data acquired by SAFIRE-A during the 23.09.1999 scientific flight. Horizontal axis indicates flight time in seconds UTC, VMR units are ppmv. Aircraft flight altitude is superimposed on the map. 
same path. The anti-correlation between Ozone and $\mathrm{ClO}$ evidences the occurrence of chlorine activation and ozone depletion processes as the aircraft approaches the vortex region. The dots superimposed on the maps indicate the exact point where the measurement was made.

\subsubsection{Measurements across a polar vortex filament}

The 10.08.1999 APE-GAIA scientific flight aimed at studying the physical and chemical conditions within and without a filament (extruded out of the polar vortex edge) predicted from high resolution Potential Vorticity (PV) fields for the 10.08.1999 between Tierra del Fuego and the Antarctic Peninsula at an altitude between 14 and $18 \mathrm{~km}$. To verify and study the occurrence of this structure a flight was planned, with a four leg track over two isentropic surfaces: $390 \mathrm{~K}$ (approximately $16 \mathrm{~km}$ ) and $450 \mathrm{~K}$ (approximately $19 \mathrm{~km}$ ). The observations carried out by the SAFIRE-A limb sounder during this flight highlighted the capability of the instrument to resolve the filament structure, with adequate spatial resolution and in good agreement with both the model expectations
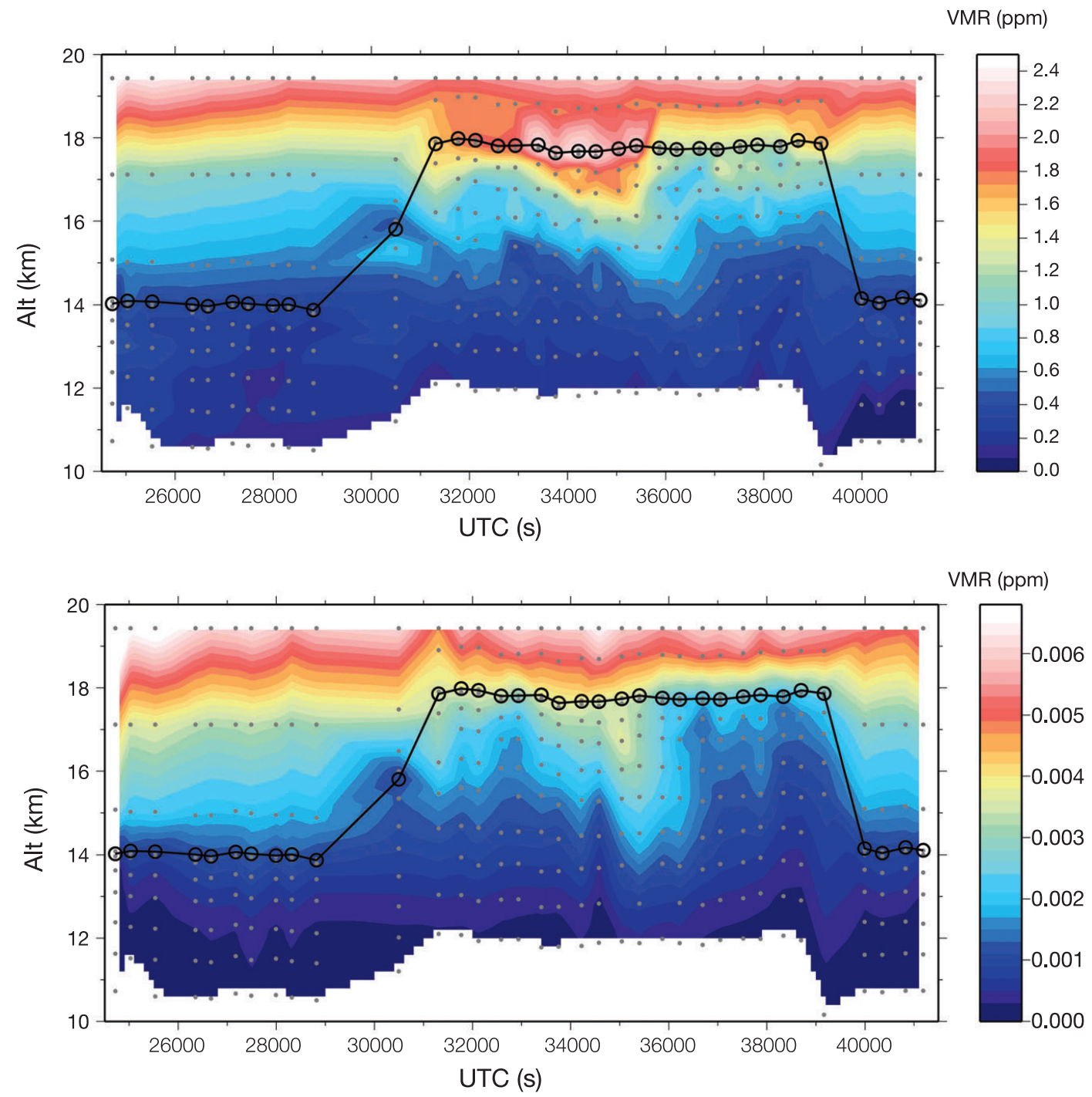

Fig. 17. Evidence of a vortex filament in simultaneous $\mathrm{VMR}$ maps of $\mathrm{O}_{3}$ (upper panel) and $\mathrm{HNO}_{3}$ (lower panel) observed during the APE-GAIA flight of 08.10.1999 near the Antarctic Peninsula 
and the measurement performed by the in situ sensors aboard the Geophysica aircraft. In fig. 17, the two dimensional distribution of $\mathrm{O}_{3}$ and $\mathrm{HNO}_{3}$ volume mixing ratio are shown.

\subsubsection{Polarization measurement on a lee wave induced PSC over the Antarctic Peninsula}

The third scientific flight of the APEGAIA mission was devoted to the study of the microphysics and chemistry inside gravity waves generated in the upper atmosphere by the interaction of surface topography and strong winds (i.e. mountain lee waves). The area between the Antarctic Peninsula and Tierra del Fuego was monitored for more than a week with local circulation models and mountain wave formation was forecast for the 10.02.1999. A flight was planned accordingly with the aim of probing the temperature perturbations and verifying the occurrence of lee-wave induced polar stratospheric clouds. The temperature experienced on the second leg of the flight, with the aircraft cruising at altitude of 18-19 km for about $800 \mathrm{~km}$, showed clearly several oscillations (with minima well down to $182 \mathrm{~K}$ ) in good agreement with the temperature fields forecasted by the model. PSC occurrence was detected by all the microphysics instruments operating onboard the M-55 aircraft (lidars and in situ instruments).

During this flight, the SAFIRE-A instrument was set up to alternate high and low spectral resolution measurements, (respectively 0.004 $\mathrm{cm}^{-1}$ and $0.03 \mathrm{~cm}^{-1}$ ). The high resolution spectra were acquired in the standard configuration of the Martin-Puplett interferometer, thus allowing the observation of vertical VMR profiles of ozone and other minor constituents. A different configuration, without input polariser was used to record the low resolution spectra. The measured quantity in this case is the difference between the vertically and the horizontally polarised components of the atmospheric signal; since the atmospheric emission is not polarized, normally this signal should be zero.

The SAFIRE-A spectrometer, in the polarization measurement configuration, observed a non zero signal that appears to be strongly time and space correlated with the polar stratospheric cloud detected by the microphysics instruments. Figure 18 shows the ratio between the difference of the two polarisation components and the horizontal component measured by the standard Martin-Puplett configuration.

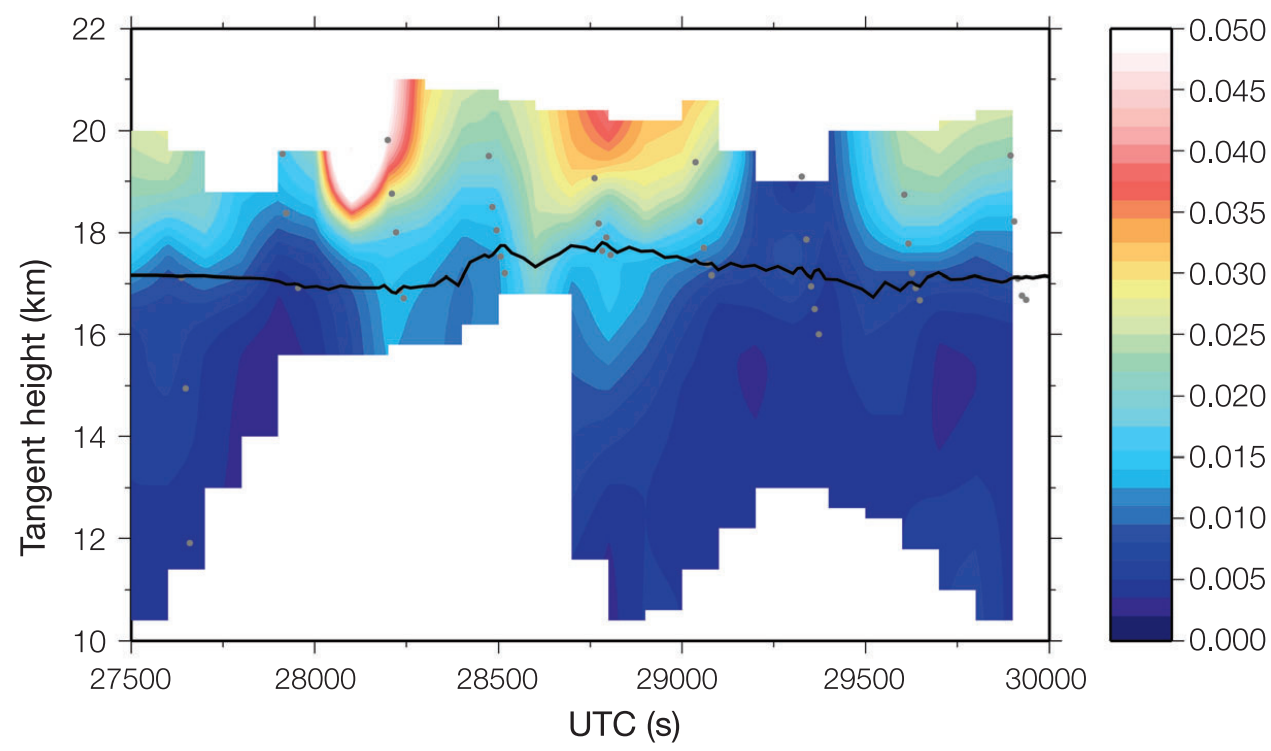

Fig. 18. Two dimensional map of the ratio between the difference of the two polarisation components and the horizontal component measured by the standard Martin-Puplett configuration (02.10.1999). 


\section{Conclusions}

Emission FT spectroscopy from airborne platform combines the intrinsic advantages of high sensitivity and selectivity of FTS instruments with the versatility of the aircraft platform, obtaining a multi-purpose tool for the characterisation of upper troposphere/ lower stratosphere air masses. Moreover, the limb-sounding observation geometry gives an optimal trade-off between resolution of vertical structures in atmospheric layering and horizontal resolution. The results obtained by the SAFIRE-A FT spectrometer during the last Antarctic campaign show the capabilities of such a combination of remotesensing instrument and airborne platform. This capabilities include determination of twodimensional volume mixing ratio maps of trace species present in concentrations ranging from ppmv to pptv, the detection of local structures due to dynamic processes in the atmosphere, and the measurement of optical properties as the polarization of atmospheric radiance. This characterizes SAFIRE-A as an instrument suitable not only for a stand-alone operation mode for the determination of atmospheric composition, but also as a powerful component of the system involving different kinds of instruments, operating both in situ and remotesensing and oriented not only to the study of atmospheric chemistry, but also of dynamic and microphysics processes.

\section{Acknowledgements}

This work was supported by the Italian Programme for Research in Antarctica (PNRAProgramma Nazionale di Ricerche in Antartide) and the Italian Space Agency (ASI-Agenzia
Spaziale Italiana), which funded most of the activities for the upgrading and utilisation of SAFIRE-A instrument. The authors wish to thank Dr. C. Lee (Queen Mary and Westfield College, London) for providing the operative support on the ${ }^{3} \mathrm{He}$-cooled detector unit.

\section{REFERENCES}

BiANCHINI, G., M. LANFRANCHI and U. CORTESI (2000): Flight qualification of a diode-laser for path difference determination of a high resolution FT spectrometer, Appl. Opt., 39, 963-966.

CARLI, B. (1989): High-resolution far-infrared FT spectroscopy of the stratosphere: optimization of the optical design of the instrument, SPIE Proc., 1145, 93-98.

CARli, B. and M CARlotTi (1992): Far-infrared and microwave spectroscopy of the Earth's atmosphere, in Spectroscopy of the Earth's Atmosphere and the Interstellar Medium, edited by K. NARAHARI RAOM and A. WEBER (Academic Press) p. 28 and following.

CARLI, B., H. FisCHER and J. PYLE (1992): Limb sounding techniques for environmental monitoring in the nineties, ESA SP-1140, Noordwijk, NL.

CARli, B., P.A.R. Ade, U. Cortesi, P. Dickinson, M. Epifani, F.C. Gannaway, A. Gignoli, C. Keim, C. Lee, C. Meny, J. Leotin, F. Mencaraglia, A.G. MurRAY, I.G. NOLT and M. RIDOLFI (1999): SAFIREA spectroscopy of the atmosphere using far-infrared emission/airborne, J. Atmos. Ocean. Technol., 16, 1313-1328.

CARlotTI, M. (1988): Global fit approach to the analysis of lim-scanning atmospheric measurements, Appl. Opt., 27, 3250-3254.

Chamberlain, J. (1978): The Principles of Interferometric Spectroscopy (John Wiles \& Sons, New York), 16-18.

Martin, D.H. and E. Puplett (1969): Polarised interferometric spectrometry for the millimetre and submillimetre spectrum, Infrared Phys., 10, 105-109.

Piesch, C., T. Gulde, C. SARTORIUS, F. Friedl-VAllon, M. SEEFELDNER, M. WOELFEL, C.E. BLOM and H. FISCHER (1996): Design of a MIPAS instrument for high-altitude aircraft, in The Second International Airborne Remote Sensing Conference and Exhibition, San Francisco, California, 24-27 June 1996, vol. II, 199-208. 